Wissenschaftliche Taschenbücher

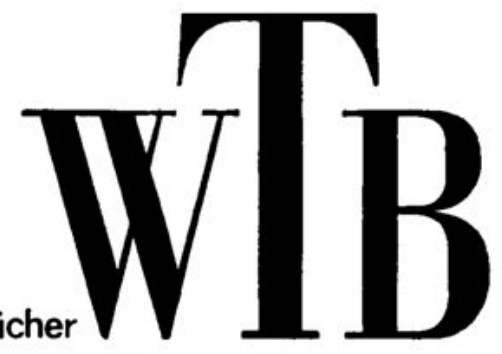

Chemie

Burkart Philipp

Gerhard Reinisch

Grundlagen

der

makromolekularen Chemie 
GUNTHER WAGNER

HANS KÜHMSTEDT

Pharmazeutische Chemie

ROLF SCHÖLLNER

Dle Oxydation organischer

Verbindungen mit Sauerstoff

ULRICH BEHRENS

MANFRED RINGPFEIL

Mikroblelle Polysaccharide

MaRTIN Finke

WALTER LEIPNITZ

Moderne Methoden der Erdölanalyse

ERICH GUNDERMaNN

Chemie und Technologie

des Braunkohlenteers

GERHARD KEMPTER

Struktur und Synthese von Vitaminen

FRIRDRICH J $\triangle \mathrm{COB}$

Bewegungsphysiologie der Pflanzen

HELLMUTH GäBLER

Forstschutz

ALFred PALISSA

Bodenzoologie in Wissenschaft, Naturhaushalt und Wirtschaft

LUDWIG SPANNHOF

Zellen und Gewebe der Tiere

\section{JOACHIM RIEMER}

Quantitative organische Mikroanalyse

ALFRED FRIESER

Mikrowellenmeßtechnik

HANS-WERNER NOUTE

Pflanzenschutz in der Landwirtschaft
WERNER HABERDITZL

Magnetochemie

HARRY PAUL

Lasertheorle, Teil I und II

D. TER HAAR

Quantentheorie

J. H. SANDERS

Die Lichtgeschwindigkelt

JRAN KUnTZMaNN

Unendliche Reihen

JEAN KUNTZMANN

Systeme von Differentialgleichungen

JEAN KUNTZManN

Komplexe Veränderliche

FERDINAND CAP

Einführung in die Plasmaphysik

I. Theoretische Grundlagen

FERdinand CaP

Einführung in die Plasmaphyslk

II. Wellen und Instabilităten

FERDINAND CAP

Einfuihrung in die Plasmaphysik

III. Magnetohydrodynamik

J. A. Rosanow

Wahrscheinlichkeitstheorle

HARRY PfEIfER

Theorie linearer Bauelemente

Elektronik für den Physiker I

HARRY PFEIFER

Die Elektronenröhre

Elektronik für den Physiker II 


\section{HARRY PFELFER}

Schaltungen mit Elektronenröhren

Elektronik für den Physiker III

\section{HARRY PFEIFER}

Leitungen und Antennen

Elektronik für den Physiker IV

\section{HARRY PFEIFER}

Mikrowellenelektronik

Elektronik für den Physiker V

HARRY PFEIFER

Halbleiterelektronik

Elektronik für den Physiker VI

\section{HARRY PFEIFER}

Schaltungen mit Transistoren

Elektronik für den Physiker VII

\section{Stephen G. BRUSh}

Kinetische Theorie, Teil I und II

\section{EBERHARD HOFMANN}

Eiweiße und Nucleinsäuren als biologische Makromoleküle

Dynamische Biochemie, Teil I

\section{EBRRHARD HOFMANN}

Enzyme und energiebereitstellende

Reaktionen des Stoffwechsels

Dynamische Biochemie, Teil II

\section{EBERHARD HOFMANN}

Der Stoffwechsel lebendiger Systeme und seine Regulation

Dynamische Biochemie, Teil III

\section{EBERHARD HOFMANN}

Grundlagen der Molekularbiologie und Regulation des Zellstoffwechsels Dynamische Biochemie, Teil.IV

\section{HERBERT GOERING}

Elementare Methoden zur Lösung von Differentialgleichungsproblemen

Pater KRUMbiegel

Isotopleeffekte

\section{M. BRINK}

\section{Kernkraffte}

DIETER ONKEN Steroide

Dieter Klada

Elementare Axiome der Mengenlehre Einführung

in die Allgemeine Mengenlehre I

GUNTER TEMBROCK

Grundlagen der Tierpsychologle

J. F. Vinson

Optische Kohärenz in der klassischen Theorie und in der Quantentheorie

W. R. HINDMARSH

Atomspektren

GUNTER TEMBROCK

Biokommunikation

Informationsübertragung

im biologischen Bereich

Teil I und II

\section{DIETER MERKeL}

Riechstoffe

John CUnninghaM

Vektoren

ERNST SCHMUTZER

Symmetrien und Erhaltungssätze der Physik

GehHARD LERCH

Pflanzenökologie

MICHAEL GÖSSEL

Angewandte Automatentheorie, Band I

MICHAEL GüSSEL

Angewandte Automatentheorie, Band II

HEINRIOH KINDLER

Der Regelkreis 
A. R. KESSEL

Akustische Kernresonanz

WOLFRAM BRAUER

HANS-WALDEMAR STREITWOLF

Theoretische Grundlagen

der Halblelterphysik

HARRY PAUL

Nichtlineare Optik, Teil I und II

DIETRIOH BENDER

ERNST-Egon PIPPIG

Einheiten - Maßsysteme - SI

Dieter KLAUa

Grundbegriffe der axdomatischen

Mengenlehre

Teil 1 und 2

Einführung

in die Allgemeine Mengenlehre II

E. G. Golstein

Konvexe Optimierung

EBERHARD TEUSCHER

Pharmakognosie, Teil I und II

SIEGFRIED HAUPTMANN

Uber den Ablauf organisch-chemischer

Reaktionen

A. S. SONIN

B. A. STRUKOW

Einführung in die Ferroelektrizität

L. A. SKORNJAKOW

Elemente der Verbandstheorie

HANS-MARTIN BARCHET

Chemie photographischer Prozesse

R. A. R. TRIOKER

Frühe Elektrodynamik

N. D. SHBWANDROW

Die Polarisation des Lichtes
A. A. SMIRnow
Metaliphysik

\section{VOLKER KEMPE}

Theorie stochastischer Systeme

ROLF BORSDORF

MANFRED SCHOLZ

Spektroskopische Methoden in der

organischen Chemie

KLAUS ODEnING

Parasitismus

Wolfgang Padli

Vierpoltheorie

und ihre Anwendung auf elektronische

Schaltungen

Dieter KLAUA

Kardinal- und Ordinalzahlen

Teil 1 und 2

Einführung

in die Allgemeine Mengenlehre III

R. A. R. TRICKER

Die Beiträge von Faraday und Maxwell zur Elektrodynamik

Hans DawczynsKI

Temperaturbeständige Faserstoffe aus organischen Polymeren

Hans Dawczynski

Temperaturbeständige Faserstoffe

aus anorganischen Polymeren

HELMUT FRIEMEL

JOSEF BROCK

Grundlagen der Immunologie

GrRaLd WOLF

Neurobiologie

HORST KeHLEN

FRANK KUSCHEL

- HoRst SACkManN

Grundlagen der chemischen Kinetik

HANS BERGNER

Tlerernährung

ULRICH SEDLAG

Blologische Schädlingsbekämpfung 


\section{MARTIN SCHMrDT \\ Pflanzenschutz Im Gartenbau}

WOLFGANG WAGNER

Chemische Thermodynamik

HANNELORE FISCHER

JOACHIM PIEHLER

Modellsysteme

der Operationsforschung

ALBERT EINSTEIN

Grundzüge der Relatlvitätstheorie

ALBERT EINSTEIN

Uber die spezielle und die allgemeine

Relativitätstheorie

MichaEL GösSRL

Wahrschelnlichkeitsautomaten

und Zufallsfolgen

HANS-JÜRGEN TREDER

Elementare Kosmologie

Peter PaUfler

DIETER LEUSCHNER

Kristallographische Grundbegriffe der

Festkörperphysik

PAUL HOFFMANN

Photosynthese

Wolfgang Meming

Kernphysikalische Elektronik

HEINZ GEMER

Ókologie der Land- und Süßwassertlere

JOACHIM NITSCHMANN

Entwicklung bei Mensch und Tier
KONRAD KREHER

Festkörperphyslk

HANS BANDEMER

ANDreas BellmanN

WOLFHART JUNG

KLAUS RICHTER

Optimale Versuchsplanung

DIETER KÖNIG

DIETRICH STOYAN

Methoden der Bedienungstheorie

KLAUS KUHNE

Werkstoff Glas

WERNER DÖPKE

Stereochemie

organischer Verblndungen

SIEGFRIED BREHMER

Einführung in die Maßtheorie

ULRICH RŐSBBERG

Determinismus und Physik

I. S. SHRLUDEW

Elektrische Kristalle

BURKART PHILIPP

GERHARD REINISCH

Grundlagen

der makromolekularen Chemie

Vorschau auf die nächsten Bände:

VOLKER KEMPE

Analyse stochastischer Systeme, Teil I

REGINE WITK0WSEI

FALKO H. HERRMANN

Einführuug in die klinische Genetik 



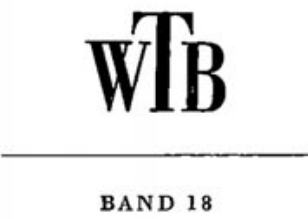

Burkart Philipp

Gerhard Reinisch

\section{Grundlagen der makromolekularen Chemie}

Mit 53 Abbildungen und 9 Tabellen 


\section{Reihe C HEM I E}

Herausgegeben im Auftrage

der Akademie der Wissenschaften der DDR

von:

Prof. Dr. H. Klare, Berlin

Prof. Dr.-Ing. Dr. h. c. E. Leibnitz, Berlin

Prof. Dr.-Ing. Dr. h. c. mult. K. Schwabe, Meinsberg

Prof. Dr. Dr. h. c. E. Thilo, Berlin

Verantwortlicher Herausgeber dieses Bandes:

Prof. Dr. H. Klare

Verfasser :

Prof. Dr. B. Philipp

Prof. Dr. G. Reinisch

Institut für Polymerenchemie

der Akademie der Wissenschaften der DDR

Teltow-Seehof

1976

2., bearbeitete Auflage

Erschienen im Akademie-Verlag, 108 Berlin, Leipziger Straße 3-4

(C) Akademie-Verlag, Berlin, 1976

Lizenznummer: $202 \cdot 100 / 483 / 76$

Herstellung: VEB Druckhaus „Maxim Gorki“, 74 Altenburg

Bestellnummer: 7600142 (7018) · LSV 1274

Printed in GDR

12,50 